\title{
Scanning Electron Microscopy for Fabrication and Imaging of Hydrogel Composites
}

\author{
Tanya Gupta ${ }^{1,2}$, Andrei Kolmakov $^{1 *}$ \\ 1. NIST, PML, Gaithersburg, MD 20899, USA; \\ 2. Maryland NanoCenter, University of Maryland, College Park, MD 20742, USA \\ * Corresponding author: andrei.kolmakov@nist.gov
}

Metal nanoparticle hydrogel composites are among the most prospective materials in catalysis [1], drug delivery [2], antimicrobial coatings [3], (bio-)sensors, soft robotics, stimuli responsive transducers etc. Synthesis of composite hydrogel can be classified into two categories: in-situ techniques are the ones where functional inclusions (e.g. functional nanoparticles) are premixed in the un-crosslinked liquid polymer solution and are entrapped in the hydrogel during the crosslinking process, whereas ex-situ techniques typically involve an impregnation process, applied post the crosslinking. In-situ encapsulation offers advantages in embedding nanoparticles homogeneously across the bulk, unlike the latter case where the distribution of nanoparticles is controlled by out-of-surface diffusion of chemicals into the gel's bulk. Here we demonstrate an in-situ method where functional metal nanoparticles become entrapped in the hydrogel network at the predefined locations via use of the e-beam induced crosslinking of liquid hydrogel composite suspension. The important advantage of our approach is an effective immobilization of the nanoparticles in the hydrogel matrix due to instant formation of solidified "cocoon" around the nanoparticle via preferential crosslinking of the near particle region by secondary and backscattered electrons (Figure 1a). These secondary electrons are thermolyzed within ca $(\sim 10 \mathrm{~nm})$ region surrounding the nanoparticle, leading to stronger irradiation dose and therefore crosslinking the polymeric cocoon around the particle

For many applications it is important to image the encapsulated particles inside the gel with high spatial resolution. Figure 1b c depicts the SEM image of the resultant encapsulated $50 \mathrm{~nm} \mathrm{Au} \mathrm{nanoparticles}$ inside the dry crosslinked hydrogel matrix collected with backscattered electrons (BSE) detector. In BSE imaging mode, the contrast of the objects is predominantly determined by the difference of the atomic numbers $(Z)$ of the nanoparticle and liquid matrix material as well as on the depth at which the electrons are collected [4]. In the SEM image Au particles with much larger effective Z compared to hydrogel matrix appear brighter, and both: their signal strength and the resolution wanes with the depth of the nanoparticle inside the gel (Figure $1 \mathrm{~b}$ ). To evaluate the feasible imaging depth for hydrogel imbedded objects we conducted Monte Carlo (MC) simulation of the BSE images of heterogeneous and compared them with the experimental data (Figure 1c). As can be seen, SEM can be used to probe nanoparticles as deep as few hundred nanometers using a $20 \mathrm{keV}$ beam energy with resolution still better compared to conventional optical microscopy.

\section{References:}

[1] N Sahiner, Progress in Polymer Science 38 (2013), p. 1329.

[2] Gaharwar, et al., Biotechnol Bioeng 111 (2014), p. 441.

[3] H Palza, International Journal of Molecular Sciences 16 (2015), p. 2099.

[4] Thiberge, et al., Review of Scientific Instruments 75 (2004), p. 2280. 


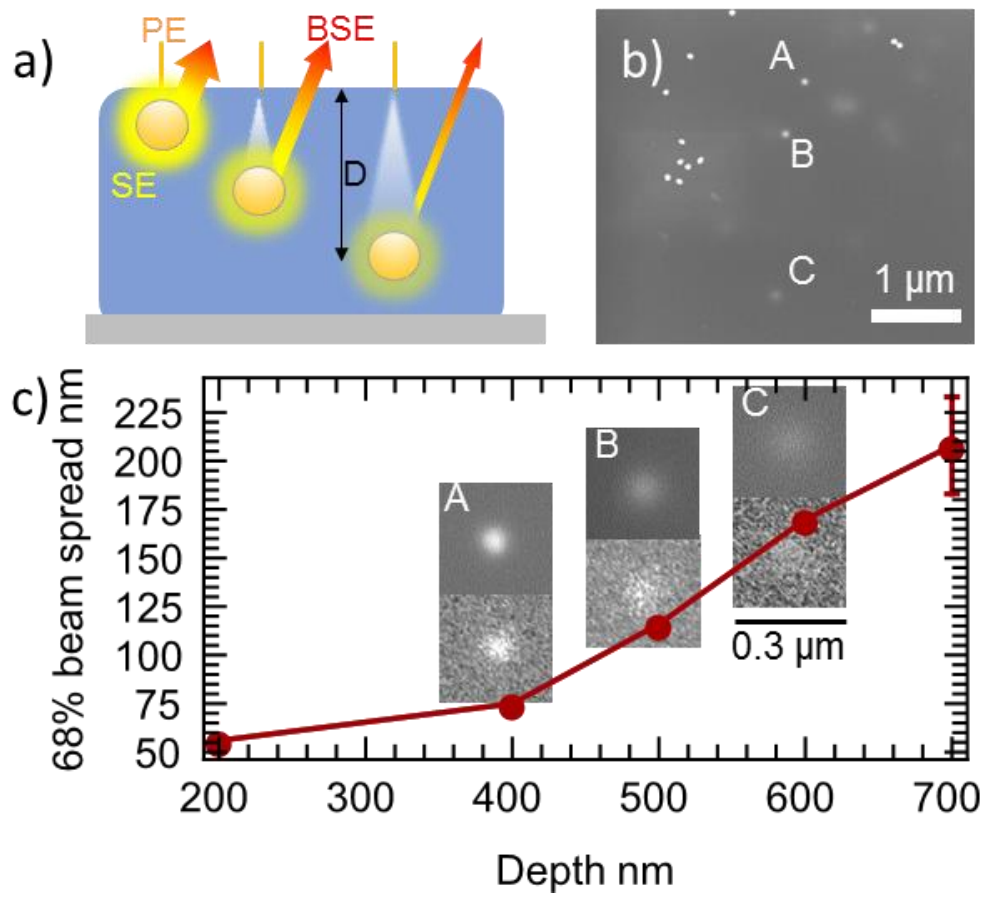

Figure 1. Detection of hydrogel imbedded nanoparticles using backscattered BSE electrons. a) Schematic representation of BSE scattering of the primary electrons from Au nanoparticles imbedded at random depths $\mathrm{D}$. At smaller $\mathrm{D}$ the value of the beam spreading is less, and BSE intensity is higher. $b$ ) BSE image of hydrogel containing $50 \mathrm{~nm}$ Au nanoparticles. c) Monte-Carlo simulation of the effective diameter of $50 \mathrm{~nm}$ Au nanoparticle based on the depth and its comparison with the particles $\mathrm{A}, \mathrm{B}$, and $\mathrm{C}$ as labelled in panel b) 